\title{
PITT'S THEOREM FOR OPERATORS BETWEEN GENERAL LORENTZ SEQUENCE SPACES
}

\author{
J. A. LÓPEZ MOLINA*
}

\begin{abstract}
We characterize the pairs of general Lorentz sequence spaces $\ell^{u, v}(v), \ell^{p, q}(\mu), 0<u, v, p, q<$ $\infty$ such that all continuous linear maps from the first space into the second one are compact.
\end{abstract}

\section{Introduction}

It is well known that a good characterization of compact operators between Banach spaces $E$ and $F$ can be used as a powerful tool for the study of the structure of these spaces. Pitt's theorem is the oldest and perhaps the most spectacular result along these lines and raises the question of finding more pairs $(E, F)$ of Banach spaces such that every continuous linear map from $E$ into $F$ is compact. Recently new pairs with this property have been found ([4], [13]) and the problem has been extended to the class of quasi Banach spaces (see [5]), because of its importance in the theory of interpolation spaces. In this paper we study Pitt's theorem between general Lorentz sequence spaces $\ell^{p, q}(\mu)$ where $0<p, q<\infty$ and $\mu$ is a general measure on $\mathrm{N}$. This study is a natural continuation (and the culmination in the case of Lorentz spaces $\left.\ell^{p, q}(\mu)\right)$ of the work developed in [5].

Given quasi Banach spaces $E$ and $F$, a continuous linear map $T: E \rightarrow F$, (an operator in the sequel for short) is said to be compact if it sends bounded subsets of $E$ into relatively compact subsets of $F$. If all operators from $E$ into $F$ are compact, we shall simply write $(E, F) \in \mathscr{K}$. The main purpose of this paper is to characterize the relation $\left(\ell^{u, v}(v), \ell^{p, q}(\mu)\right) \in \mathscr{K}$.

Section 2 contains basic facts about Lorentz sequence spaces and some preparatory results which we shall need in the following sections. In section 3 we prove some structural propositions concerning the behaviour of sequences and operators on Lorentz sequence spaces and we prove the main theorem of the paper. The symbol $\|\cdot\|_{E}$ will be the quasinorm on a given quasi Banach

\footnotetext{
* This research is partially supported by the DGICYT, project PB97-0333.

Received February 18, 1999; in revised form April 1, 2000.
} 
space $E$. We shall say that $\left\{z_{n}\right\}_{n=1}^{\infty} \subset E$ is a seminormalized sequence in $E$ if it is bounded and there is a number $\varepsilon>0$ such that $\left\|z_{n}\right\|_{E} \geq \varepsilon$ for every $n \in \mathrm{N}$.

\section{Basic facts on Lorentz sequence spaces}

Let $(\Omega, \Sigma, \mu)$ be a $\sigma$-finite measure space. Let $\mathscr{M}(\Omega, \mu)$ denote the collection of all extended scalar-valued (real or complex) $\Sigma$-measurable functions on $\Omega$ and which are finite $\mu$-a.e. The distribution function $\mu_{f}$ of a function $f$ in $\mathscr{M}(\Omega, \mu)$ is given by

$$
\mu_{f}(\lambda):=\mu\{x \in \Omega|| f(x) \mid>\lambda\}, \quad(\lambda \geq 0) .
$$

The decreasing rearrangement of $f \in \mathscr{M}(\Omega, \mu)$ is the function $f_{\mu}^{*}$ defined on $[0, \infty[$ by

$$
f_{\mu}^{*}(t):=\inf \left\{\lambda \geq 0 \mid \mu_{f}(\lambda) \leq t\right\},(t \geq 0) .
$$

Suppose $1<p<\infty, 1 \leq q<\infty$. The Lorentz space $L^{p, q}(\Omega, \mu)$ consists of all $f$ in $\mathscr{M}(\Omega, \mu)$ for which the quantity

$$
\|f\|_{\mu, p, q}:=\left(\int_{0}^{\infty}\left(t^{1 / p} f^{*}(t)\right)^{q} \frac{d t}{t}\right)^{1 / q}
$$

is finite. It can be proved that $\|\cdot\|_{\mu, p, q}$ is a norm if $1 \leq q \leq p$ (see for instance Chapter 4, Theorem 4.3 in [2]). However we shall not need this fact but only that $\|\cdot\|_{\mu, p, q}$ is a quasinorm for every $0<p, q<\infty$. Indeed, since the decreasing rearrangement of the sum of two functions has the property

$$
\forall t>0, \quad(f+g)^{*}(t) \leq f^{*}\left(\frac{t}{2}\right)+g^{*}\left(\frac{t}{2}\right)
$$

(see Chapter 2, Proposition 1.7 in [2]), with an easy change of variable and Hölder's inequality with exponents $\frac{1}{q}$ and $\frac{1}{1-q}$ if $q<1$, we get

$$
\|f+g\|_{\mu, p, q} \leq \max \left(2^{\frac{1}{p}}, 2^{\frac{1}{p}+\frac{1}{q}-1}\right)\left(\|f\|_{\mu, p, q}+\|g\|_{\mu, p, q}\right) .
$$

An important consequence of (2) is the $r$-normability of $L^{p, q}(\mu)$ where $2^{\frac{1}{r}}=\max \left(2^{1+\frac{1}{p}}, 2^{\frac{1}{p}+\frac{1}{q}}\right)$ (Aoki-Rolewicz's theorem, see for instance Theorem 1.3 in [7]), i.e. there is in $L^{p, q}(\mu)$ an equivalent quasinorm $\|\cdot\|_{\mu,(p, q)}$ such that

$$
\forall f, g \in L^{p, q}(\mu), \quad\|f+g\|_{\mu,(p, q)}^{r} \leq\|f\|_{\mu,(p, q)}^{r}+\|g\|_{\mu,(p, q)}^{r} .
$$

We refer the reader to [2] and [10] for more details on Lorentz spaces.

From now on, we shall work only with the particular case $\Omega=\mathrm{N}$ and a purely atomic measure $\mu$ on $N$ defined by $\left.\mu(\{i\})=\mu_{i} \in\right] 0, \infty[$ for every 
$i \in \mathrm{N}$. Then $L^{p, q}(\mathrm{~N}, \mu)$ is a Lorentz sequence space which will be denoted by $\ell^{p, q}(\mu)$ or simply by $\ell^{p, q}$ if $\mu_{i}=1$ for every $i \in \mathrm{N}$. If there is no risk of confusion, we shall write $\|\cdot\|_{p, q}$ instead of $\|\cdot\|_{\mu, p, q}$, or $\|\cdot\|_{\ell p, q}(\mu)$ if we want to call attention to the space.

Let $\rho=\left\{\rho_{i}\right\}_{i=1}^{\infty}$ be a sequence of strictly positive numbers. In some instances we shall need weighted spaces

$$
\ell^{p, q}(\rho, \mu):=\left\{\left(x_{i}\right) \mid\left(x_{i} \rho_{i}\right) \in \ell^{p, q}(\mu)\right\} .
$$

In such cases, the canonical quasinorm of $\left(x_{i}\right) \in \ell^{p, q}(\rho, \mu)$ is $\left\|\left(x_{i} \rho_{i}\right)\right\|_{\ell p, q}(\mu)$. Given $\alpha>0$, the symbol $\mu^{\alpha}$ will denote the sequence $\left(\mu_{i}^{\alpha}\right)$. Analogously, we define $\rho / \mu:=\left(\rho_{i} / \mu_{i}\right), 1 / \rho:=\left(1 / \rho_{i}\right)$ and $\rho \mu:=\left(\rho_{i} \mu_{i}\right)$.

For every $i \in \mathrm{N}$, the sequence $(0,0, \ldots, 1,0,0, \ldots)$ with 1 in the $i$-th place will always be denoted by the symbol $e_{i}$ and $P_{i}: \ell^{p, q}(\mu) \longrightarrow \mathrm{K}$ will be the canonical projection from $\ell^{p, q}(\mu)$ onto the scalar field $\mathrm{K}$ where each sequence is projected onto its $\mathrm{i}$-th component.

In order to make concrete computations we shall need an explicit formula for the quasinorm $\|x\|_{p, q}$ of a sequence $x=\left(x_{i}\right) \in c_{0} \cap \ell^{p, q}(\mu)$ with respect to the measure $\mu=\left(\mu_{i}\right)$ on $\mathrm{N}$. Let $\sigma: \mathrm{N} \rightarrow \mathrm{N}$ be such that

$$
\left|x_{\sigma(1)}\right|=\max \left\{\left|x_{n}\right| \mid n \in \mathrm{N}\right\},
$$

and if $i>1$,

$$
\left|x_{\sigma(i)}\right|=\max \left\{\left|x_{n}\right| \mid n \notin\{\sigma(1), \sigma(2), \ldots, \sigma(i-1)\}\right\} .
$$

Then, the decreasing rearrangement of $x$ is the function $x^{*}(t)$ given by

$$
x^{*}(t)=\left|x_{\sigma(1)}\right| \text { if } t \in\left[0, \mu_{\sigma(1)}[\right.
$$

and

$$
\forall i>1, \quad x^{*}(t)=\left|x_{\sigma(i)}\right| \text { if } t \in\left[\sum_{j=1}^{i-1} \mu_{\sigma(j)}, \sum_{j=1}^{i} \mu_{\sigma(j)}[\right.
$$

and its quasinorm is

$$
\|x\|_{p, q}=\left(\frac{p}{q} \sum_{i=1}^{\infty}\left|x_{\sigma(i)}\right|^{q}\left(\left(\sum_{j=1}^{i} \mu_{\sigma(j)}\right)^{\frac{q}{p}}-\left(\sum_{j=1}^{i-1} \mu_{\sigma(j)}\right)^{\frac{q}{p}}\right)\right)^{\frac{1}{q}},
$$

where $\sum_{j=1}^{0} \mu_{\sigma(j)}$ is defined to be equal to 0 , and hence

$$
\|x\|_{p, q}=\left(\frac{p}{q} \sum_{i=1}^{\infty}\left|x_{\sigma(i)}\right|^{q}\left(\sum_{j=1}^{i} \mu_{\sigma(j)}\right)^{\frac{q}{p}}\left(1-\left(1-\frac{\mu_{\sigma(i)}}{\sum_{j=1}^{i} \mu_{\sigma(j)}}\right)^{\frac{q}{p}}\right)\right)^{\frac{1}{q}} .
$$


Since the function $F(\alpha):=\left(1-(1-\alpha)^{\frac{q}{p}}\right) \alpha^{-1}$ is strictly positive, monotone and continuous in $] 0,1]$ and $\lim _{\alpha \rightarrow 0} F(\alpha)=\frac{q}{p}$, we have $\min \left(1, \frac{q}{p}\right) \leq F(\alpha) \leq$ $\max \left(1, \frac{q}{p}\right)$ for every $\left.\left.\alpha \in\right] 0,1\right]$. If we define for every $x \in \ell^{p, q}(\mu) \cap c_{0}$

$$
\||x|\|_{p, q}:=\left(\sum_{i=1}^{\infty}\left|x_{\sigma(i)}\right|^{q} \mu_{\sigma(i)}\left(\sum_{j=1}^{i} \mu_{\sigma(j)}\right)^{\frac{q}{p}-1}\right)^{\frac{1}{q}}
$$

we have that

$$
p \leq q \Longrightarrow\left(\frac{p}{q}\right)^{\frac{1}{q}}\|\| x\left\|_{p, q} \leq\right\| x\left\|_{p, q} \leq\right\|\|x \mid\|_{p, q}
$$

and

$$
p>q \Longrightarrow\|x\|\left\|_{p, q} \leq\right\| x\left\|_{p, q} \leq\left(\frac{p}{q}\right)^{\frac{1}{q}}\right\| x\|\|_{p, q} .
$$

Then $\|\mid \cdot\|_{p, q}$ is a quasinorm equivalent to $\|.\|_{p, q}$ on $\ell^{p, q}(\mu) \cap c_{0}$, which will be more easy to use in some problems. Since $\|.\|_{p, q}$ is monotone in $\ell^{p, q}(\mu)$, (10)

$\forall x, y \in \ell^{p, q}(\mu) \cap c_{0}, \quad|x| \leq|y| \Longrightarrow \begin{cases}\|x\|_{p, q} \leq\left(\frac{p}{q}\right)^{\frac{1}{q}}\|\mid y\|_{p, q} & \text { if } q \leq p \\ \|x\|_{p, q} \leq\left(\frac{q}{p}\right)^{\frac{1}{q}}\|\mid y\|_{p, q} & \text { if } q \geq p .\end{cases}$

The strict inclusions

$$
\ell^{p, q} \subset \ell^{u, v} \quad \forall 0<q, v<\infty \quad \forall 0<p<u<\infty
$$

and

$$
\ell^{p, q} \subset \ell^{p, v} \quad \forall 0<q \leq v<\infty \quad \forall 0<p<\infty
$$

are well known (see [2] and [14]) and will be used in the sequel. Unfortunately these inclusions are not true for arbitrary measures $\mu$ in N. For instance, if $\mu=$ $\left(\mu_{i}\right)$ verifies $\lim _{i \rightarrow \infty} \mu_{i}=0$ and $u<p$, if the inclusion $\ell^{u, v}(\mu) \subset \ell^{p, q}(\mu)$ were true, it would be continuous by the closed graph theorem. However

$$
\forall i \in \mathrm{N}\left\|\frac{e_{i}}{\mu_{i}^{\frac{1}{u}}}|\||_{\ell^{u, v}(\mu)}=1 \text { but } \lim _{i \rightarrow \infty}\right\| \frac{e_{i}}{\mu_{i}^{\frac{1}{u}}} \|\left.\right|_{\ell^{p, q}(\mu)}=\lim _{i \rightarrow \infty} \frac{1}{\mu_{i}^{\frac{1}{u}-\frac{1}{p}}}=\infty
$$

which is a contradiction. This situation will be a source of complications in our future developments. 
The next technical lemma on pairwise disjoint elements of a Lorentz sequence space will be basic:

Lemma 2.1. Let $\left\{z^{n}\right\}_{n=1}^{\infty}$ be a sequence of elements of $\ell^{p, q}(\mu)$ of finite pairwise disjoint supports and let $\left\{a_{n}\right\}_{n=1}^{\infty} \subset \mathrm{K}^{\mathrm{N}}$. Let $r:=\min \{p, q\}$ and $s:=\max \{p, q\}$.

1) If there is $M>0$ such that $\left\|z^{n}\right\|_{p, q} \leq M$ for every $n \in \mathrm{N}$, then

$$
\forall n, \in \mathrm{N}\left\|\sum_{i=1}^{n} a_{i} z^{i}\right\|_{p, q} \leq M\left(\sum_{i=1}^{n}\left|a_{i}\right|^{r}\right)^{\frac{1}{r}} .
$$

2) If there is $\varepsilon>0$ such that $\left\|z^{n}\right\|_{p, q} \geq \varepsilon$ for every $n \in \mathrm{N}$, then

$$
\forall n, \in \mathrm{N}\left\|\sum_{i=1}^{n} a_{i} z^{i}\right\|_{p, q} \geq \varepsilon\left(\sum_{i=1}^{n}\left|a_{i}\right|^{s}\right)^{\frac{1}{s}} .
$$

Proof. Both statements are inmediate consequences of the known fact that $\ell^{p, q}(\mu)$ satisfies an upper $r$-estimate and a lower $s$-estimate (see for instance [8] for a more explicit proof).

The next definition will be useful in order to simplify both the exposition and the proofs of our results. Let $\left\{z^{n}\right\}_{n=1}^{\infty}:=\left\{\left(z_{i}^{n}\right)\right\}_{n=1}^{\infty}$ be a sequence in $\ell^{p, q}(\mu)$.

Definition 2.2. a) A sequence of type

$$
w^{m}=\sum_{i=n_{m-1}+1}^{n_{m}} z_{i}^{k_{m}} e_{i} \quad \forall m \in \mathrm{N},
$$

where $\left\{k_{m}\right\}_{m=1}^{\infty}$ and $\left\{n_{m}\right\}_{m=0}^{\infty}$ are strictly increasing sequences in $\mathrm{N}$ with $k_{1}=$ $1, n_{0}=0$, will be called a diagonal subsequence of consecutive linear sections of $\left\{z^{n}\right\}_{n=1}^{\infty}$.

b) A sequence of type

$$
w^{m}=\sum_{i \in S_{m}} z_{i}^{k_{m}} e_{i} \quad \forall m \in \mathrm{N},
$$

where $S_{m} \subset\left[n_{m-1}+1, n_{m}\right] \subset \mathrm{N}$ for every $m \in \mathrm{N}$, will be called a diagonal subsequence of general sections of $\left\{z^{n}\right\}_{n=1}^{\infty}$.

c) We say that $\left\{z^{n}\right\}_{n=1}^{\infty}$ is a coordinatewise null sequence if

$$
\forall i \in \mathrm{N}, \quad \lim _{n \rightarrow \infty} z_{i}^{n}=0 .
$$


COROLlary 2.3. Let $\left\{z^{n}\right\}_{n=1}^{\infty}$ be a coordinatewise null seminormalized sequence in $\ell^{p, q}(\mu)$. that

1) Let $s:=\max \{p, q\}$. There are $M_{1}>0$ and a subsequence $\left\{z^{n_{k}}\right\}_{k=1}^{\infty}$ such

(15) $\forall n \in \mathrm{N}, \quad \forall a_{1}, a_{2}, \ldots, a_{n} \in \mathrm{K}, \quad\left\|\sum_{k=1}^{n} a_{k} z^{n_{k}}\right\|_{p, q} \geq M_{1}\left(\sum_{k=1}^{n}\left|a_{k}\right|^{q}\right)^{\frac{1}{q}}$.

2) Let $r:=\min \{p, q\}$. There are $M_{2}>0$ and a subsequence $\left\{z^{n_{k}}\right\}_{k=1}^{\infty}$ such that

(16) $\forall n \in \mathrm{N}, \quad \forall a_{1}, a_{2}, \ldots, a_{n} \in \mathrm{K}, \quad\left\|\sum_{k=1}^{n} a_{k} z^{n_{k}}\right\|_{p, q} \leq M_{2}\left(\sum_{k=1}^{n}\left|a_{k}\right|^{q}\right)^{\frac{1}{q}}$.

Proof. Let $t>0$ be such that there is an equivalent $t$-norm $\|$.$\| on \ell^{p, q}(\mu)$ and $\alpha>0$ satisfying

$$
\forall x \in \ell^{p, q}(\mu), \quad \alpha\|x\| \leq\|x\|_{p, q} \leq \frac{1}{\alpha}\|x\| .
$$

Suppose that

$$
\forall n \in \mathrm{N}, \quad 0<\varepsilon \leq\left\|z^{n}\right\|_{p, q} \leq M
$$

Since

$$
\forall i \in \mathrm{N}, \quad \lim _{n \rightarrow \infty} z_{i}^{n}=0
$$

by (18), starting with $k_{1}=1, n_{0}=0$ and using the method of gliding hump, we obtain two strictly increasing sequences of natural numbers $\left(k_{m}\right)_{m=1}^{\infty}$ and $\left(n_{m}\right)_{m=0}^{\infty}$, such that, putting

$$
\begin{gathered}
f^{1}=0, \quad \forall m \geq 2, \quad f^{m}:=\sum_{i=1}^{n_{m-1}} z^{k_{m}} e_{i} \in \ell^{p, q}(\mu) \\
\forall m \geq 1, \quad u^{m}:=\sum_{i=n_{m-1}+1}^{n_{m}} z_{i}^{k_{m}} e_{i} \in \ell^{p, q}(\mu)
\end{gathered}
$$

and

$$
\forall m \geq 1, \quad g^{m}:=z^{k_{m}}-f^{m}-u^{m} \in \ell^{p, q}(\mu),
$$


we have

$$
\forall m \geq 1, \quad \forall h \geq n_{m}, \quad\left\|\sum_{i=n_{m-1}+1}^{h} z_{i}^{k_{m}} e_{i}\right\|_{p, q} \geq \frac{\varepsilon}{2}
$$

$$
\begin{aligned}
& \forall m \geq 2, \quad\left\|f^{m}\right\|_{p, q}^{t} \leq \frac{1}{\alpha^{t}} \sum_{i=1}^{n_{m-1}}\left|z_{i}^{k_{m}}\right|^{t}\left\|e_{i}\right\|_{p, q}^{t} \leq \frac{\varepsilon^{t} \alpha^{2 t}}{2^{m+2 t+1}+2^{t} \alpha^{2 t}}, \\
& \forall m \geq 1, \quad\left\|g^{m}\right\|_{p, q}^{t}=\left\|\sum_{i=n_{m}+1}^{\infty} z_{i}^{k_{m}} e_{i}\right\|_{p, q}^{t} \leq \frac{\varepsilon^{t} \alpha^{2 t}}{2^{m+2 t+1}+2^{t} \alpha^{2 t}}
\end{aligned}
$$

1) For every $n \in \mathrm{N}$ and every finite sequence $\left(a_{i}\right)_{i=1}^{n} \subset \mathrm{K}^{n}$, by (17), (20), Lemma 2.1.2, (21) and (22) we have

$$
\begin{aligned}
&\left\|\sum_{i=1}^{n} a_{i} z^{k_{i}}\right\|_{p, q}^{t} \geq \alpha^{t}\left\|\sum_{i=1}^{n} a_{i} f^{i}+\sum_{i=1}^{n} a_{i} u^{i}+\sum_{i=1}^{n} a_{i} g^{i}\right\|^{t} \\
& \geq \alpha^{t}\left\|\sum_{i=1}^{n} a_{i} u^{i}\right\|^{t}-\alpha^{t}\left\|\sum_{i=1}^{n} a_{i} f^{i}\right\|^{t}-\alpha^{t}\left\|\sum_{i=1}^{n} a_{i} u^{i}\right\|^{t} \\
& \geq\left(\frac{\varepsilon}{2}\right)^{t} \alpha^{2 t}\left(\sum_{i=1}^{n}\left|a_{i}\right|^{s}\right)^{\frac{t}{s}}-2 \varepsilon^{t} \alpha^{2 t} \sum_{i=1}^{n} \frac{\left|a_{i}\right|^{t}}{2^{i+2 t+1}} \\
&=\left(\frac{\varepsilon}{2}\right)^{t} \alpha^{2 t}\left(\sum_{i=1}^{n}\left|a_{i}\right|^{s}\right)^{\frac{t}{s}} \\
& \quad-\varepsilon^{t} \alpha^{2 t}\left(\sum_{i=1}^{n}\left|a_{i}\right|^{s}\right)^{\frac{t}{s}}\left(\sum_{i=1}^{n} \frac{\left|a_{i}\right|^{t}}{\left(\sum_{i=1}^{n}\left|a_{i}\right|^{s}\right)^{\frac{t}{s}} \frac{1}{2^{i+2 t}}}\right) \\
& 2^{2 t}\left(\sum_{i=1}^{n}\left|a_{i}\right|^{s}\right)^{\frac{t}{s}}
\end{aligned}
$$

which proves our assertion.

2) The proof is analogous using Lemma 2.1.1.

To end this introductory section we need to mention the fundamental relation between Lorentz spaces and interpolation spaces. Given quasi Banach spaces $E_{i}, i=0,1$, such that each $E_{i}$ is continuously embedded in some Hausdorff topological vector space $E$, we consider on $E_{0}+E_{1}$ the quasinorm defined by

$$
\|x\|_{E_{0}+E_{1}}=\inf \left\{\left\|x_{0}\right\|_{E_{0}}+\left\|x_{1}\right\|_{E_{1}} \mid x=x_{0}+x_{1}, x_{0} \in E_{0}, x_{1} \in E_{1}\right\} .
$$


The classical theory of the real interpolation method of Banach spaces (see [1] and [3]) has been extended by Sagher (see [16]) to the case of quasi Banach spaces. Since we shall never use the explicit definition of the quasinorm in such interpolated quasi Banach spaces $\left(E_{0}, E_{1}\right)_{\theta, q}, 0<\theta<1,0<q \leq \infty$, we simply refer the interested reader to the original paper [16]. However, we shall use next a theorem which is part of a more general important result of Freitag (see [6]).

Theorem 2.4 (Freitag, [6]). Let $0<\theta<1,0<p<\infty$, $0<q<\infty$ and $0<p_{0}<p_{1}<\infty$ satisfying the equation $\frac{1}{p}=\frac{1-\theta}{p_{0}}+\frac{\theta}{p_{1}}$. The following isomorphisms hold by means of the identity map:

a) $\ell^{p, q}(\mu) \approx\left(\ell^{p(1-\theta)}(\mu), \ell^{\infty}(\mu)\right)_{\theta, q} \approx\left(\ell^{p_{0}}(\mu), \ell^{p_{1}}(\mu)\right)_{\theta, q}$.

b) Given strictly positive weights $\rho:=\left(\rho_{i}\right), \eta:=\left(\eta_{i}\right)$ we have

$$
\left(\ell^{p(1-\theta)}(\rho, \mu), \ell^{\infty}(\eta, \mu)\right)_{\theta, q} \approx \ell^{p, q}\left(\eta,\left(\frac{\rho}{\eta}\right)^{p(1-\theta)} \mu\right) .
$$

c) For every $0<\eta<1$,

$$
\left(\ell^{p(1-\eta)}(\mu), \ell^{\infty}(\mu)\right)_{\eta, \infty} \approx\left(\ell^{p_{0}}(\mu), \ell^{p_{1}}(\mu)\right)_{\theta, \infty} .
$$

This theorem gives us some information about Lorentz sequence spaces. For instance, an easy consequence is that every sectional subspace of $\ell^{p, q}(\mu)$ is again another Lorentz space $\ell^{p, q}(v)$. Also, by elementary considerations, we obtain from Theorem 2.4:

Proposition 2.5. $\left\{e_{i}\right\}_{i=1}^{\infty}$ is a Schauder basis in every space $\ell^{p, q}(\mu)$.

Another application of Theorem 2.4 concerns duality. Given $\ell^{p, q}(\mu)$, choose a number $0<\theta<1$ such that $p(1-\theta)<1$. By Theorem 2.4, $\ell^{p, q}(\mu)$ is isomorphic to $\left(\ell^{p(1-\theta)}(\mu), \ell^{\infty}(\mu)\right)_{\theta, q}$. Since the normed dual of $\ell^{s}, 0<s \leq 1$ is $\ell^{\infty}$, denoting by $\mu_{0}$ the ordinary measure on $\mathrm{N}$, we have the isometries

$$
\left(\ell^{p(1-\theta)}(\mu)\right)^{\prime}=\left(\ell^{p(1-\theta)}\left(\mu^{\frac{1}{p(1-\theta)}}, \mu_{0}\right)\right)^{\prime}=\ell^{\infty}\left(\mu^{1-\frac{1}{p(1-\theta)}}, \mu\right)
$$

with duality bilinear form given by

$$
\forall\left(x_{i}\right) \in \ell^{p(1-\theta)}(\mu), \quad \forall\left(y_{i}\right) \in \ell^{\infty}\left(\mu^{1-\frac{1}{p(1-\theta)}}, \mu\right) \quad\left\langle\left(x_{i}\right),\left(y_{i}\right)\right\rangle=\sum_{i=1}^{\infty} x_{i} y_{i} \mu_{i} .
$$


With exactly the same method of proof used in [1], pages 59-65, and using Theorem 2.4, if $q \leq 1$ we can prove the isomorphisms (24)

$$
\begin{aligned}
\left(\ell^{p, q}(\mu)\right)^{\prime} \approx\left(\ell^{p(1-\theta)}(\mu), \ell^{\infty}(\mu)\right)_{\theta, q}^{\prime} & \approx\left(\ell^{\infty}\left(\mu^{1-\frac{1}{p(1-\theta)}}, \mu\right), \ell^{1}(\mu)\right)_{\theta, \infty} \\
& =\left(\ell^{1}(\mu), \ell^{\infty}\left(\mu^{1-\frac{1}{p(1-\theta)}}, \mu\right)\right)_{1-\theta, \infty}
\end{aligned}
$$

with the same duality pairing given by (23), and analogously

$$
\left(\ell^{p, q}(\mu)\right)^{\prime \prime} \approx \ell^{\frac{1}{1-\theta}, 1}\left(\mu^{\frac{1}{p(1-\theta)}-1}, \mu^{\frac{1}{p(1-\theta)}}\right) .
$$

Arguing in the same way with obvious changes if $q>1$, we obtain (25)

$$
\left(\ell^{p, q}(\mu)\right)^{\prime} \approx\left(\ell^{p(1-\theta)}(\mu), \ell^{\infty}(\mu)\right)_{\theta, q}^{\prime} \approx\left(\ell^{1}(\mu), \ell^{\infty}\left(\mu^{1-\frac{1}{p(1-\theta)}}, \mu\right)\right)_{1-\theta, \frac{q}{q-1}}
$$

and in all cases

$$
\left(\ell^{p, q}(\mu)\right)^{\prime \prime} \approx \ell^{\frac{1}{1-\theta}, \bar{q}}\left(\mu^{\frac{1}{p(1-\theta)}-1}, \mu^{\frac{1}{p(1-\theta)}}\right)
$$

where $\bar{q}=q$ if $q>1$, and $\bar{q}=1$ if $q \leq 1$.

We shall often need to find subspaces isomorphic to $\ell^{q}$ in certain concrete special situations. Our main tool here will be an extension to the quasi Banach spaces setting of a classical theorem by Levy in [9] which has been obtained in [11]:

Theorem 2.6. Let $0<q<\infty$ and let $E_{i}, i=0,1$ be quasi Banach spaces. Let $\left\{x_{n}\right\}_{n=1}^{\infty}$ be a seminormalized sequence in the interpolation space $\left(E_{0}, E_{1}\right)_{\theta, q}$, such that lim $m_{n \rightarrow \infty} x_{n}=0$ in $E_{0}+E_{1}$. Then there is a subsequence $\left\{x_{n_{k}}\right\}_{k=1}^{\infty}$ such that its closed linear span in $\left(E_{0}, E_{1}\right)_{\theta, q}$ is isomorphic to $\ell^{q}$.

Levy's result has been improved by Brudnyi and Krugljak in Theorem 4.6.22) of [3], where it is shown that a Banach space of type $\ell^{p, q}(\mu)$ has complemented subspaces isomorphic to $\ell^{q}$. The extension of this result to the general quasinormed case is given in [12]:

Proposition 2.7. Every Lorentz sequence space $\ell^{p, q}(\mu)$ has a complemented subspace isomorphic to $\ell^{q}$.

\section{On necessary conditions}

Lemma 3.1. If $v \leq q$ then $\left(\ell^{u, v}(v), \ell^{p, q}(\mu)\right) \notin \mathscr{K}$ holds.

Proof. By Proposition 2.7, $\ell^{v}$ and $\ell^{q}$ are complemented subspaces of $\left(\ell^{u, v}(v)\right.$ and $\left.\ell^{p, q}(\mu)\right)$ respectively. The proof follows from the classical Pitt's theorem. 
Lemma 3.2. Let the measures $v=\left(v_{i}\right)$ and $\mu=\left(\mu_{i}\right)$ on $\mathrm{N}$ be such that the sequences $\left\{v_{i}\right\}_{i=1}^{\infty}$ and $\left\{\mu_{i}\right\}_{i=1}^{\infty}$ have some adherent point in $] 0, \infty[$. If $u<p$, then $\left(\ell^{u, v}(v), \ell^{p, q}(\mu)\right) \notin \mathscr{K}$.

Proof. There are $\alpha>0, \beta>0$ in R and infinite subsets $J_{v}, J_{\mu}$ of N such that

$$
\forall n \in J_{n u}, \forall m \in J_{\mu} \quad \alpha \leq v_{n} \leq \beta \quad \text { and } \quad \alpha \leq \mu_{m} \leq \beta
$$

Projecting onto suitable complemented sectional subspaces, we can assume that $J_{v}=J_{\mu}=\mathrm{N}$. Given $\left(\alpha_{i}\right) \in \ell^{u, v}(v)$ and $n \in \mathrm{N}$, if $\sigma:\{1,2, \ldots, n\} \longrightarrow \mathrm{N}$ is an injective map giving the decreasing rearrangement of the finite sequence $\left(\alpha_{i}\right)_{i=1}^{n}$, we have for $u \leq v$

$$
\begin{aligned}
\frac{1}{\beta^{\frac{1}{u}-\frac{1}{v}}}\left(\sum_{i=1}^{n}\left|\alpha_{\sigma(i)}\right|^{v}\left(\sum_{j=1}^{i} v_{\sigma(j)}\right)^{\frac{v}{u}-1}\right)^{\frac{1}{v}} & \leq\left(\sum_{i=1}^{n}\left|\alpha_{\sigma(i)}\right|^{v} i^{\frac{v}{u}-1}\right)^{\frac{1}{v}} \\
& \leq \frac{1}{\alpha^{\frac{1}{u}-\frac{1}{v}}}\left(\sum_{i=1}^{n}\left|\alpha_{\sigma(i)}\right|^{v}\left(\sum_{j=1}^{i} v_{\sigma(j)}\right)^{\frac{v}{u}-1}\right)^{\frac{1}{v}} .
\end{aligned}
$$

We can make a similar computation in the case $u>v$. These inequalities with Proposition 2.5 show that $\ell^{u, v}(v)$ is isomorphic to $\ell^{u, v}$. Analogously, $\ell^{p, q}(\mu)$ is isomorphic to $\ell^{p, q}$. Now, as $u<p$ the inclusion map $\ell^{u, v} \subset \ell^{p, q}$ is not compact.

LeMmA 3.3. Let $v=\left(v_{i}\right)$ and $\mu=\left(\mu_{i}\right)$ be measures in $\mathrm{N}$ such that $\left\{v_{i}\right\}_{i=1}^{\infty}$ has a finite strictly positive adherent point, $\lim _{i \rightarrow \infty} \mu_{i}=0$ and $\sum_{i=1}^{\infty} \mu_{i}=\infty$. If $u<p$ we have that

a) $\left(\ell^{u, v}(v), \ell^{p, q}(\mu)\right) \notin \mathscr{K}$ and

b) $\left(\ell^{u, v}(\mu), \ell^{p, q}(v)\right) \notin \mathscr{K}$.

Proof. As in Lemma 3.2, projecting onto suitable complemented sectional subspaces of $\ell^{u, v}(v)$ and $\ell^{p, q}(\mu)$ we can assume that

$$
\forall i \in \mathrm{N}, \quad 0<\mu_{i+1} \leq \mu_{i}<1
$$

and that $\ell^{u, v}(v)$ and $\ell^{p, q}(v)$ are identically isomorphic to $\ell^{u, v}$ and $\ell^{p, q}$ respectively.

By the hypothesis on $\mu$, the sequence $\left\{n_{k}\right\}_{k=0}^{\infty}$ such that $n_{0}:=0$ and

$$
\forall k \geq 1, \quad n_{k}=\min \left\{h \in \mathrm{N} \mid \sum_{i=n_{k-1}+1}^{h} \mu_{i} \geq 1\right\}
$$


is well defined. From this definition we obtain

$$
\forall k \in \mathrm{N}, \quad 1 \leq \sum_{i=n_{k-1}+1}^{n_{k}} \mu_{i}=\sum_{i=n_{k-1}+1}^{n_{k}-1} \mu_{i}+\mu_{n_{k}}<1+\mu_{n_{k}} \leq 2 .
$$

Let $J_{k}:=\left\{i \in \mathrm{N} \mid n_{k-1}+1 \leq i \leq n_{k}\right\}$ for every $k \in \mathrm{N}$ and let $u<$ $s<p$. By Theorem 2.4, given a number $0<\theta<1$, the spaces $\ell^{u, v}, \ell^{s, q}$ and $\ell^{p, q}(\mu)$ are isomorphic to the spaces $\left(\ell^{(1-\theta) u}, \ell^{\infty}\right)_{\theta, v},\left(\ell^{(1-\theta) s}, \ell^{\infty}\right)_{\theta, q}$ and $\left(\ell^{(1-\theta) p}(\mu), \ell^{\infty}(\mu)\right)_{\theta, q}$ respectively.

a) We consider the map $T: \ell^{(1-\theta) s}+\ell^{\infty} \longrightarrow \ell^{(1-\theta) p}(\mu)+\ell^{\infty}(\mu)$ defined by the rule $T\left(\left(\alpha_{i}\right)\right):=\left(\beta_{i}\right)$ where

$$
\forall\left(\alpha_{i}\right) \in \ell^{(1-\theta) s}+\ell^{\infty}, \quad \forall i, k \in \mathrm{N}, \quad \beta_{i}=\alpha_{k} \text { if } i \in J_{k} .
$$

By (27) we have

$$
\begin{aligned}
\forall\left(\alpha_{i}\right) \in \ell^{(1-\theta) s}, \quad\left\|T\left(\left(\alpha_{i}\right)\right)\right\|_{\ell^{(1-\theta) p}(\mu)} & =\left(\sum_{k=1}^{\infty}\left|\alpha_{k}\right|^{(1-\theta) p} \sum_{i \in J_{k}} \mu_{i}\right)^{\frac{1}{(1-\theta) p}} \\
& \leq 2\left\|\left(\alpha_{i}\right)\right\|_{\ell^{(1-\theta) p}} \leq 2\left\|\left(\alpha_{i}\right)\right\|_{\ell^{(1-\theta) s}}
\end{aligned}
$$

and

$$
\forall\left(\alpha_{i}\right) \in \ell^{\infty}, \quad\left\|T\left(\left(\alpha_{i}\right)\right)\right\|_{\ell^{\infty}(\mu)} \leq\left\|\left(\alpha_{i}\right)\right\|_{\ell^{\infty}} .
$$

Then, by the interpolation theorem (see [16]), $T$ is continuous from $\left(\ell^{(1-\theta) s}\right.$, $\left.\ell^{\infty}\right)_{\theta, q}$ into $\left(\ell^{(1-\theta) p}(\mu), \ell^{\infty}(\mu)\right)_{\theta, q}$.

Now, let $J$ be the inclusion $\ell^{u, v} \subset \ell^{s, q}$. We have $\left\|e_{i}\right\|_{u, v}=1$ for each $i \in \mathrm{N}$ but

$\forall i \in \mathrm{N}, \quad\left\|T J\left(e_{i}\right)\right\|_{\ell^{p, q}(\mu)}^{p}=\left\|\sum_{j=n_{i-1}+1}^{n_{i}} e_{j}\right\|_{\ell^{p, q}(\mu)}^{p}=\left(\frac{p}{q}\right)^{\frac{p}{q}} \sum_{j=n_{i-1}+1}^{n_{i}} \mu_{j} \geq\left(\frac{p}{q}\right)^{\frac{p}{q}}$

and $\lim _{i \rightarrow \infty} P_{j} T J\left(e_{i}\right)=0$ for every $j \in \mathrm{N}$. Hence $T J$ is not compact.

b) By Lemma 3.1, we can suppose moreover that $v>q$. By Proposition 2.7, $\ell^{v}$ is a complemented topological subspace of $\ell^{u, v}(\mu)$. Moreover, if $v<p$, we have the continuous inclusion $\ell^{v} \subset \ell^{p, q}$ and hence $\left(\ell^{u, v}(v), \ell^{p, q}(\mu)\right) \notin \mathscr{K}$. Hence we only need to consider the case $u<p \leq v, q<v$.

b.1) Suppose $u \leq 1$. Choose a number $0<\theta<1$ such that $p(1-$ $\theta)<1$. By Theorem 2.4, $\ell^{u, v}(\mu)$ is isomorphic to the interpolation space $\left(\ell^{u(1-\theta)}(\mu), \ell^{\infty}(\mu)\right)_{\theta, v}$. Define for later use $\bar{w}=w$ if $w>1$ and $\bar{w}=1$ if $w \leq 1$. 
Now we consider the map $S$ from $\mathrm{K}^{\mathrm{N}}$ into $\mathrm{K}^{\mathrm{N}}$ defined by

$$
\forall\left(\alpha_{i}\right) \in \mathrm{K}^{\mathrm{N}}, \quad S\left(\left(\alpha_{i}\right)\right)=\left(\sum_{i \in J_{k}} \alpha_{i} \mu_{i}^{\frac{1}{u(1-\theta)}}\right)_{k=1}^{\infty} .
$$

Choose a number $r$ such that $u<r<p$. Since $r(1-\theta)>u(1-\theta)$, the restriction of $S$ to $\ell^{u(1-\theta)}(\mu)$ is continuous from this space into $\ell^{r(1-\theta)}$. To see this compute

$$
\begin{aligned}
\left(\sum_{k=1}^{\infty}\left|\sum_{i \in J_{k}} \alpha_{i} \mu_{i}^{\frac{1}{u(1-\theta)}}\right|^{r(1-\theta)}\right)^{\frac{1}{r(1-\theta)}} & \leq\left(\sum_{k=1}^{\infty}\left|\sum_{i \in J_{k}} \alpha_{i} \mu_{i}^{\frac{1}{u(1-\theta)}}\right|^{u(1-\theta)}\right)^{\frac{1}{u(1-\theta)}} \\
& \leq\left(\sum_{k=1}^{\infty} \sum_{i \in J_{k}}\left|\alpha_{i}\right|^{u(1-\theta)} \mu_{i}\right)^{\frac{1}{u(1-\theta)}} \\
& =\left\|\left(\alpha_{i}\right)\right\|_{\ell^{u(1-\theta)}(\mu)},
\end{aligned}
$$

where we need the fact that $u(1-\theta)<1$. On the other hand, by (27) we have

$$
\sum_{i \in J_{k}} \mu_{i}^{\frac{1}{u(1-\theta)}} \leq \sum_{i \in J_{k}} \mu_{i} \leq 2
$$

and hence $S$ is clearly continuous from $\ell^{\infty}(\mu)$ into $\ell^{\infty}$. By the interpolation property, (see [16]) $S$ is continuous from $\left(\ell^{u(1-\theta)}(\mu), \ell^{\infty}(\mu)\right)_{\theta, v}$ into $\left(\ell^{r(1-\theta)}, \ell^{\infty}\right)_{\theta, v}$ and hence from $\ell^{u, v}(\mu)$ into $\ell^{r, v}$.

Let $J$ be the inclusion $\ell^{r, v} \subset \ell^{p, q}$. Let $R: \ell^{\frac{1}{1-\theta}, \bar{v}}\left(\mu^{\frac{1}{u(1-\theta)}}\right) \longrightarrow \ell^{u, v}(\mu)^{\prime \prime}$ be the canonical isomorphism given by (recall (26))

$$
\forall\left(\alpha_{i}\right) \in \ell^{\frac{1}{1-\theta}, \bar{v}}\left(\mu^{\frac{1}{u(1-\theta)}}\right), \quad R\left(\left(\alpha_{i}\right)\right)=\left(\alpha_{i} \mu_{i}^{1-\frac{1}{u(1-\theta)}}\right) .
$$

Then $J^{\prime \prime} S^{\prime \prime} R$ is not compact. In fact, by (1), for each $k \in \mathrm{N}$, we have

$$
\begin{aligned}
\left\|\sum_{i \in J_{k}} e_{i}\right\|_{\ell \frac{1}{(1-\theta), 1}\left(\mu \frac{1}{\mu(1-\theta)}\right)}=\frac{1}{(1-\theta)}\left(\sum_{i \in J_{k}} \mu_{i}^{\frac{1}{u(1-\theta)}}\right)^{(1-\theta)} & \leq \frac{1}{1-\theta} \sum_{i \in J_{k}} \mu_{i}^{\frac{1}{u}} \\
& \leq \frac{1}{1-\theta} \sum_{i \in J_{k}} \mu_{i} \\
& \leq \frac{2}{1-\theta} .
\end{aligned}
$$


However, applying (26) to the space $\ell^{p, q}$ we obtain

$$
\begin{aligned}
\left\|J^{\prime \prime} S^{\prime \prime} R\left(\sum_{i \in J_{k}} e_{i}\right)\right\|_{\ell^{\frac{1}{1-\theta}, \bar{q}}}^{\frac{1}{1-\theta}}=\left\|\left(\sum_{i \in J_{k}} \mu_{i}\right) e_{k}\right\|_{\ell^{\frac{1}{1-\theta}, \bar{q}}}^{\frac{1}{1-\theta}} & =\left(\frac{1}{(1-\theta) \bar{q}}\right)^{\frac{1}{(1-\theta) \bar{q}}} \sum_{i \in J_{k}} \mu_{i} \\
& \geq\left(\frac{1}{(1-\theta) \bar{q}}\right)^{\frac{1}{(1-\theta) \bar{q}}} .
\end{aligned}
$$

As a consequence, since the transposed map of a compact operator is also compact, $J S: \ell^{u, v}(\mu) \longrightarrow \ell^{p, q}$ is not compact.

b.2) If $1<u$, choosing $u<r<p$, and defining the number $0<\theta<1$ such that $1=(1-\theta) u<(1-\theta) r$, by Theorem 2.4 and the interpolation property of operators (see [16]) we can show in the same way as above that the map $\bar{S}$ given by

$$
\forall\left(\alpha_{i}\right) \in \ell^{u, v}(\mu), \quad \bar{S}\left(\left(\alpha_{i}\right)\right)=\left(\sum_{i \in J_{k}} \alpha_{i} \mu_{i}\right)_{k=1}^{\infty}
$$

is continuous from $\ell^{u, v}(\mu)$ into $\ell^{r, v}$ and that $J \bar{S}$ is not compact.

\section{Main results}

Proposition 4.1. Let $r:=\min \{u, v\}$. Assume $q \leq p \leq r$. Let $T$ be an operator from $\ell^{u, v}(v)$ into $\ell^{p, q}(\mu)$. Assume $\left\{z^{n}\right\}_{n=1}^{\infty} \subset \ell^{u, v}(v)$ and $\left\{T\left(z^{n}\right)\right\}_{n=1}^{\infty} \subset \ell^{p, q}(\mu)$ are coordinatewise null seminormalized sequences. Then there is a subsequence of $\left\{T\left(z^{n}\right)\right\}_{n=1}^{\infty}$ which is equivalent to the standard unit basis of $\ell^{q}$.

Proof. If $p=q$ the conclusion is easy by consecutive application of corollaries 2.3.1 and 2.3.2.

Hence we assume $q<p$. There is a number $\theta \in] 0,1[$ such that $q=$ $(1-\theta) p$. By Theorem 2.4, $\ell^{p, q}(\mu)$ is isomorphic to $\left(\ell^{q}(\mu), \ell^{\infty}(\mu)\right)_{\theta, q}$. Then, by Theorem 2.6, it is enough to show that $\left\{v^{n}\right\}_{n=1}^{\infty}:=\left\{T\left(z^{n}\right)\right\}_{n=1}^{\infty}$ has a subsequence convergent to 0 in $\ell^{q}(\mu)+\ell^{\infty}(\mu)$.

By the hypothesis on $\left\{v^{n}\right\}_{n=1}^{\infty}$, starting with $s_{1}=1, r_{0}=0$ and using the standard gliding hump argument, we can determine $\varepsilon>0$ and a diagonal subsequence of consecutive linear sections

$$
\left\{\gamma^{h}\right\}_{h=1}^{\infty}:=\left\{\sum_{i=r_{h-1}+1}^{r_{h}} v_{i}^{s_{h}} e_{i}\right\}_{h=1}^{\infty}
$$


generated by two strictly increasing subsequences $\left\{r_{h}\right\}_{h=0}^{\infty}$ and $\left\{s_{h}\right\}_{h=1}^{\infty}$ in N such that

$$
\forall h \geq 2, \quad\left\|\sum_{i=1}^{r_{h-1}} v_{i}^{s_{h}} e_{i}\right\|_{p, q} \leq \frac{\varepsilon}{2^{h+2}}
$$

and

(29) $\forall h \geq 1, \quad\left\|\sum_{i=r_{h-1}+1}^{r_{h}} v_{i}^{s_{h}} e_{i}\right\|_{p, q} \geq \frac{\varepsilon}{2}$ and $\left\|\sum_{i=r_{h}+1}^{\infty} v_{i}^{s_{h}} e_{i}\right\|_{p, q} \leq \frac{\varepsilon}{2^{h+2}}$.

Hence, by the continuity of the inclusion map $\ell^{p, q}(\mu) \subset \ell^{q}(\mu)+\ell^{\infty}(\mu)$, the proof will be finished if we show that $\lim _{h \rightarrow \infty} \gamma^{h}=0$ in $\ell^{q}(\mu)+\ell^{\infty}(\mu)$.

For every $h \in \mathrm{N}$ and $\delta>0$ we define $J_{h}:=\left\{i \in \mathrm{N} \mid r_{h-1}+1 \leq i \leq r_{h}\right\}$, $P_{h, \delta}:=\left\{i \in J_{h}|| v_{i}^{s_{h}} \mid \leq \delta\right\}, G_{h, \delta}=J_{k} \backslash P_{k, \delta}$ and

$$
\forall h \in \mathrm{N}, \quad y^{h, \delta}:=\sum_{i \in G_{h, \delta}} v_{i}^{s_{h}} e_{i} .
$$

We now check that

$$
\lim _{h \rightarrow \infty}\left\|y^{h, \delta}\right\|_{\ell^{q}(\mu)}=0 .
$$

If this were not the case, there would be $\rho>0$ such that for some subsequence, again denoted by $\left\{\gamma^{h}\right\}_{h=1}^{\infty}$,

$$
\forall h \in \mathrm{N}, \quad\left(\sum_{i \in G_{h, \delta}}\left|v_{i}^{s_{h}}\right|^{q} \mu_{i}\right)^{\frac{1}{q}} \geq \rho .
$$

Since the set $\left\{\gamma^{h} \mid h \in \mathrm{N}\right\}$ is bounded, denoting by $\left|G_{h, \delta}\right|$ the cardinal number of the set $G_{h, \delta}$, and using (10), (7) and (31), we would have for some fixed $K>0$ and some injective map $\sigma:\left[1,\left|G_{h, \delta}\right|\right] \subset \mathrm{N} \longrightarrow \mathrm{N}$

$$
\begin{aligned}
K \geq\left(\frac{p}{q}\right)^{\frac{1}{q}}\left\|\left|\gamma^{h}\right|\right\|_{p, q} & \geq\left\|\sum_{i \in G_{h, \delta}} v_{i}^{s_{h}} e_{i} \mid\right\|_{p, q}=\left(\sum_{i=1}^{\left|G_{h, \delta}\right|} \frac{\left|v_{\sigma(i)}^{s_{h}}\right|^{q} \mu_{\sigma(i)}}{\left(\sum_{j=1}^{i} \mu_{\sigma(j)}\right)^{1-\frac{q}{p}}}\right)^{\frac{1}{q}} \\
& \geq\left(\sum_{i=1}^{\left|G_{h, \delta}\right|} \frac{\left|v_{\sigma(i)}^{s_{h}}\right|^{q} \mu_{\sigma(i)}}{\left(\sum_{j \in G_{h, \delta}} \mu_{j}\right)^{1-\frac{q}{p}}}\right)^{\frac{1}{q}} \geq \frac{\rho}{\left(\sum_{j \in G_{h, \delta}} \mu_{j}\right)^{\frac{1}{q}-\frac{1}{p}}} .
\end{aligned}
$$


Hence

$$
\sum_{j \in G_{h, \delta}} \mu_{j} \geq\left(\frac{\rho}{K}\right)^{\frac{q p}{p-q}}
$$

On the other hand we have

$$
K \geq\left(\sum_{i=1}^{\left|G_{h, \delta}\right|} \frac{\left|v_{\sigma(i)}^{s_{h}}\right|^{q} \mu_{\sigma(i)}}{\left(\sum_{j=1}^{i} \mu_{\sigma(j)}\right)^{1-\frac{q}{p}}}\right)^{\frac{1}{q}} \geq \delta\left(\sum_{i=1}^{\left|G_{h, \delta}\right|} \frac{\mu_{\sigma(i)}}{\left(\sum_{j \in G_{h, \delta}} \mu_{j}\right)^{1-\frac{q}{p}}}\right)^{\frac{1}{q}}=\delta\left(\sum_{j \in G_{h, \delta}} \mu_{j}\right)^{\frac{1}{p}}
$$

and hence

$$
\sum_{j \in G_{h, \delta}} \mu_{j} \leq\left(\frac{K}{\delta}\right)^{p}
$$

The strict inclusion $\ell^{r, q} \subset \ell^{r, r}=\ell^{r}$, holds. Since $\ell^{p, q} \subset \ell^{r, q}$, there exists a strictly decreasing sequence $\left(\alpha_{i}\right) \in \ell^{r} \backslash \ell^{p, q}$. Hence

$$
\sum_{i=1}^{\infty} \frac{\left|\alpha_{i}\right|^{q}}{i^{1-\frac{q}{p}}}=\infty
$$

By Lemma 2.1.1, the set $\left\{\sum_{h=1}^{n} \alpha_{h} z^{s_{h}} \mid n \in \mathrm{N}\right\}$ is bounded in $\ell^{u, v}(v)$. Hence, by (28) and (29), the set $\left\{\sum_{h=1}^{n} \alpha_{h} \gamma^{s_{h}} \mid h \in \mathrm{N}\right\}$ is bounded in $\ell^{p, q}(\mu)$. Afortiori $\left\{\sum_{h=1}^{n} \alpha_{h} y^{h, \delta} \mid h \in \mathrm{N}\right\}$ will be bounded in $\ell^{p, q}(\mu)$. However, using (7), (10), (33) and (34) we have

(36)

$$
\begin{aligned}
\left(\frac{p}{q}\right)^{\frac{1}{q}}\left\|\left|\sum_{h=1}^{n} \alpha_{h} y^{h, \delta}\right|\right\|_{p, q} & \geq \delta\left\|\left|\sum_{h=1}^{n} \alpha_{h} \sum_{i \in G_{h, \delta}} e_{i}\right|\right\|_{p, q} \\
& \geq \delta\left(\sum_{h=1}^{n}\left|\alpha_{h}\right|^{q} \sum_{i \in G_{h, \delta}} \frac{\mu_{i}}{\left(\sum_{m=1}^{h} \sum_{j \in G_{m, \delta}} \mu_{j}\right)^{1-\frac{q}{p}}}\right)^{\frac{1}{q}} \\
& \geq \delta\left(\sum_{h=1}^{n}\left|\alpha_{h}\right|^{q} \frac{\sum_{i \in G_{h, \delta}} \mu_{i}}{h^{1-\frac{q}{p}}\left(\frac{K}{\delta}\right)^{p-q}}\right)^{\frac{1}{q}} \\
& \geq \delta\left(\frac{\rho}{K}\right)^{\frac{p}{p-q}}\left(\frac{\delta}{K}\right)^{\frac{p}{q}-1}\left(\sum_{h=1}^{n} \frac{\left|\alpha_{h}\right|^{q}}{h^{1-\frac{q}{q}}}\right)^{\frac{1}{q}}
\end{aligned}
$$

which is unbounded with $n$ by (35), which contradicts the continuity of $T$. 
As a consequence, given $\eta>0$ there is $h_{0} \in \mathrm{N}$ such that for every $h \geq h_{0}$

$$
\left\|\gamma^{h}\right\|_{\ell q}(\mu)+\ell^{\infty}(\mu) \leq\left\|\sum_{i \in G_{h, \frac{\eta}{2}}} v_{i}^{s_{h}} e_{i}\right\|_{\ell^{q}(\mu)}+\left\|\sum_{i \in P_{h, \frac{\eta}{2}}} v_{i}^{s_{h}} e_{i}\right\|_{\ell^{\infty}(\mu)} \leq \frac{\eta}{2}+\frac{\eta}{2}=\eta
$$

and the proposition is proved.

Lemma 4.2. Every seminormalized coordinatewise null sequence in $\ell^{p, q}(\mu)$ has a subsequence equivalent to the canonical basis of $\ell^{q}$ in each of the following situations:

a) If the measure $\mu=\left(\mu_{n}\right)$ in $\mathrm{N}$ satisfies $\lim _{n \rightarrow \infty} \mu_{n}=\infty$.

b) If $\mu(\mathrm{N})<\infty$.

Proof. Let $\left\{w^{m}\right\}_{m=1}^{\infty}:=\left\{\left(w_{i}^{m}\right)\right\}_{m=1}^{\infty} \subset \ell^{p, q}(\mu)$ be a seminormalized coordinatewise null sequence such that $\left\|w^{m}\right\|_{p, q} \leq K$ for every $m \in \mathrm{N}$ and some $K>0$. Choose a number $0<\theta<1$. By Theorem 2.4, $\ell^{p, q}(\mu)$ is isomorphic to $\left(\ell^{(1-\theta) p}(\mu), \ell^{\infty}(\mu)\right)_{\theta, q}$. Then, by Theorem 2.6 it is enough to find a subsequence $\left\{w^{m_{k}}\right\}_{k=1}^{\infty}$ such that $\lim _{k \rightarrow \infty} w^{m_{k}}=0$ in $\ell^{(1-\theta) p}(\mu)+\ell^{\infty}(\mu)$. With the method and notations of Corollary 2.3, we choose a diagonal subsequence of consecutive linear sections $\left\{u^{m}\right\}_{m=1}^{\infty}$ of $\left\{w^{m}\right\}_{m=1}^{\infty}$ determined by certain strictly increasing subsequences $\left\{k_{m}\right\}_{m=1}^{\infty},\left\{n_{m}\right\}_{m=0}^{\infty}$ in N and satisfying (20), (21) and (22). Then we only have to show that $\left\{u^{m}\right\}_{m=1}^{\infty}$ has a subsequence convergent to 0 in $\ell^{(1-\theta) p}(\mu)+\ell^{\infty}(\mu)$.

a) Given $\varepsilon>0$ there is $i_{0} \in \mathrm{N}$ such that

$$
\forall i \geq i_{0}, \quad \forall m \in \mathrm{N} \quad K \geq\left\|w^{m}\right\|_{p, q} \geq\left\|w_{i}^{m} e_{i}\right\|_{p, q}=\left(\frac{p}{q}\right)^{\frac{1}{q}}\left|w_{i}^{m}\right| \mu_{i}^{\frac{1}{p}} \geq \frac{\left|w_{i}^{m}\right|}{\varepsilon} .
$$

Since $\left\{w^{m}\right\}_{m=1}^{\infty}$ is coordinatewise null, this means that $\lim _{m \rightarrow \infty} w^{m}=0$ in $\ell^{\infty}(\mu)$ and hence in $\ell^{(1-\theta) p}(\mu)+\ell^{\infty}(\mu)$ too.

b) Choose a number $0<\theta<1$ such that $(1-\theta) p<q$. Since $\mu(\mathbb{N})<\infty$, we have

$$
\left(\ell^{(1-\theta) p}(\mu), \ell^{\infty}(\mu)\right)_{\theta, q} \subset \ell^{p(1-\theta)}(\mu)+\ell^{\infty}(\mu)=\ell^{p(1-\theta)}(\mu) .
$$

Now it is enough to show that $\lim _{m \rightarrow \infty} u^{m}=0$ in $\ell^{p(1-\theta)}(\mu)$. If this were not the case, there would be an $\varepsilon>0$ such that for some subsequence, again denoted by $\left\{u^{m}\right\}_{m=1}^{\infty}$, the inequality $\left\|u^{m}\right\|_{\ell^{p(1-\theta)}(\mu)} \geq \varepsilon$ holds for every $m \in \mathrm{N}$. If $r:=\min \{p, q\}$, selecting a sequence $\left(\alpha_{m}\right) \in \ell^{r} \backslash \ell^{p(1-\theta)}$, by Lemma 2.1, the set $\left\{\sum_{i=1}^{m} \alpha_{i} w^{i} \mid m \in \mathrm{N}\right\}$ will be bounded in $\ell^{p, q}(\mu)$ but it will be unbounded 
in $\ell^{(1-\theta) p}(\mu)$ since

$$
\begin{aligned}
\left\|\sum_{k=1}^{m} \alpha_{k} u^{k}\right\|_{\ell^{(1-\theta) p}(\mu)} & =\left(\sum_{k=1}^{m}\left|\alpha_{k}\right|^{(1-\theta) p}\left\|u^{k}\right\|_{\ell^{(1-\theta) p}(\mu)}^{(1-\theta) p}\right)^{\frac{1}{(1-\theta) p}} \\
& \geq \varepsilon\left(\sum_{k=1}^{m}\left|\alpha_{k}\right|^{(1-\theta) p}\right)^{\frac{1}{(1-\theta) p}}
\end{aligned}
$$

but this contradicts (37).

Proposition 4.3. Suppose $p \leq q \leq u<v$, and $T \in \mathscr{L}\left(\ell^{u, v}(v), \ell^{p, q}(\mu)\right)$. Let $\left\{z^{n}\right\}_{n=1}^{\infty}$ be a coordinatewise null bounded sequence in $\ell^{u, v}(v)$ such that $\left\{T\left(z^{n}\right)\right\}_{n=1}^{\infty}$ is a coordinatewise null seminormalized sequence in $\ell^{p, q}(\mu)$. Then there is a diagonal subsequence $\left\{\gamma^{n_{k}}\right\}_{k=1}^{\infty}$ of general sections of $\left\{z^{n}\right\}_{n=1}^{\infty}$ which is equivalent to the standard unit basis of $\ell^{v}$ and such that $\left\{T\left(\gamma^{n_{k}}\right)\right\}_{k=1}^{\infty}$ is a seminormalized coordinatewise null sequence in $\ell^{p, q}(\mu)$.

Proof. By the continuity of $T,\left\{z^{n}\right\}_{n=1}^{\infty}$ is also seminormalized in $\ell^{u, v}(v)$. Using the gliding hump method as in Corollary 2.3, we find a subsequence $\left\{u^{m}\right\}_{m=1}^{\infty}$ of consecutive linear sections of $\left\{z^{n}\right\}_{n=1}^{\infty}$ satisfying inequalities (20), (21) and (22). As a consequence, by the triangle inequality of $t$-norms and the continuity of projections $P_{i}, i \in \mathrm{N}$, it is easy to see that $\left\{T\left(u^{m}\right)\right\}_{m=1}^{\infty}$ is also a seminormalized and coordinatewise null sequence in $\ell^{p, q}(\mu)$. Hence we can assume that the given sequence $\left\{z^{n}\right\}_{n=1}^{\infty}$ is already a sequence of consecutive linear sections of a bounded sequence.

Let $\left\{n_{h}\right\}_{h=0}^{\infty}$ and $\left\{k_{h}\right\}_{h=1}^{\infty}$ be strictly increasing sequences in $\mathrm{N}$ with $n_{0}=$ $0, k_{1}=1$ and let $\varepsilon>0, M>0$ in $\mathrm{R}$ be such that

$$
\forall h \in \mathrm{N}, \quad \varepsilon<\left\|T\left(z^{h}\right)\right\|_{p, q} \leq\|T\|\left\|z^{h}\right\|_{u, v}:=\|T\|\left\|_{i=n_{h-1}+1}^{n_{h}} z_{i}^{k_{h}} e_{i}\right\|_{u, v} \leq M .
$$

This implies that $\|T\|>0$. Put $J_{h}:=\left\{i \in \mathrm{N} \mid n_{h-1}+1 \leq i \leq n_{h}\right\}$ for every $h \in \mathrm{N}$. Fix a number $k>0$ such that $k u>1$. For every $h \in \mathrm{N}$ and $\delta>0$ we define $P_{h, \delta}:=\left\{i \in J_{h}|| z_{i}^{k_{h}} \mid \leq \delta\right\}, G_{h, \delta}=\left\{i \in J_{h}|\delta<| z_{i}^{k_{h}} \mid \leq \frac{1}{\delta^{k}}\right\}$ and

$$
\forall h \in \mathrm{N}, \quad y^{h, \delta}:=\sum_{i \in G_{h, \delta}} z_{i}^{k_{h}} e_{i} \text { and } w^{h, \delta}:=\sum_{i \in J_{h} \backslash\left(G_{h, \delta} \cup P_{h, \delta}\right)} z_{i}^{k_{h}} e_{i} .
$$

Then, by (38), (1), (4) and (5) we have

$$
\frac{M}{\|T\|} \geq\left\|y^{h, \delta}\right\|_{u, v} \geq \delta\left(\frac{u}{v}\right)^{\frac{1}{v}}\left(\sum_{i \in G_{h, \delta}} v_{i}\right)^{\frac{1}{u}}
$$


and hence

$$
\forall h \in \mathrm{N}, \quad \sum_{i \in G_{h, \delta}} v_{i} \leq C_{\delta}:=\left(\frac{M}{\|T\| \delta}\right)^{u}\left(\frac{v}{u}\right)^{\frac{u}{v}} .
$$

We now check that $\left(\beta_{i}^{h}\right):=\left\{T\left(y^{h, \delta}\right)\right\}_{h=1}^{\infty}$ is coordinatewise null in $\ell^{p, q}(\mu)$. The set

$$
R:=\left\{\sum_{h=1}^{n} \alpha_{h} y^{h, \delta} \mid\left\|\left(\alpha_{h}\right)\right\|_{\ell^{u, v}} \leq 1\right\}
$$

is bounded in $\ell^{u, v}(v)$ since by (7), (10) and (39), if $\sigma:\{1,2, \ldots, n\} \longrightarrow \mathrm{N}$ is an injective mapping giving the decreasing rearrangement of $\left(\alpha_{i}\right)_{i=1}^{n}$, we have (40)

$$
\begin{aligned}
\forall n \in \mathrm{N},\|\| \sum_{h=1}^{n} \alpha_{h} y^{h, \delta}\|\|_{u, v}^{v} & \leq \frac{1}{\delta^{k v}} \frac{v}{u} \sum_{h=1}^{n} \sum_{i \in G_{\sigma(h), \delta}}\left|\alpha_{\sigma(h)}\right|^{v} v_{i}\left(\sum_{j=1}^{h} \sum_{m \in G_{\sigma(j), \delta}} v_{m}\right)^{\frac{v}{u}-1} \\
& \leq \frac{1}{\delta^{k v}} \frac{v}{u} C_{\delta}^{\frac{v}{u}-1} \sum_{h=1}^{n}\left|\alpha_{\sigma(h)}\right|^{v} h^{\frac{v}{u}-1} \sum_{i \in G_{\sigma(h), \delta}} v_{i} \\
& \leq \frac{1}{\delta^{k v}} \frac{v}{u} C_{\delta}^{\frac{v}{u}} \sum_{h=1}^{n}\left|\alpha_{\sigma(h)}\right|^{v} h^{\frac{v}{u}-1} \\
& <\infty .
\end{aligned}
$$

Fix $i \in \mathrm{N}$. Then, by boundedness of $R$

$$
\begin{aligned}
\forall n \in \mathrm{N} \sup _{\left\|\left(\alpha_{h}\right)\right\|_{\ell, u} \leq 1}\left|\sum_{h=1}^{n} \alpha_{h} \beta_{i}^{h}\right| & =\sup _{\left\|\left(\alpha_{h}\right)\right\|_{\ell \mu, v} \leq 1}\left|P_{i} T\left(\sum_{h=1}^{n} \alpha_{h} y^{h, \delta}\right)\right| \\
& \leq \sup _{\omega \in R}\left|P_{i} T(\omega)\right|:=\rho<\infty .
\end{aligned}
$$

This means that the sequence $\left(\beta_{i}^{h}\right)_{h=1}^{\infty}$ defines a continuous linear form on $\ell^{u, v}$. By (24) and (25), $\left(\ell^{u, v}\right)^{\prime}$ is isomorphic to $\left(\ell^{1}, \ell^{\infty}\right)_{1-\theta, \bar{v}}$, where $\bar{v}=v /(v-1)$ if $v>1$ and $\bar{v}=\infty$ if $v \leq 1$. By Theorem 2.4, this space is isomorphic to $\left(\ell^{p_{0}}, \ell^{p_{1}}\right)_{\chi, \bar{v}} \subset \ell^{p_{1}}$ where $0<p_{0}<p_{1}<\infty, 0<\chi<1$ and $\theta=(1-$ $\chi) / p_{0}+\chi / p_{1}$. Hence, by (23), we obtain $\left(\beta_{i}^{h}\right)_{h=1}^{\infty} \in \ell^{p_{1}}$, and $\lim _{h \rightarrow \infty} \beta_{i}^{h}=0$ and $\left\{T\left(y^{h, \delta}\right)\right\}_{h=1}^{\infty}$ is coordinatewise null in $\ell^{p, q}(\mu)$.

Let us see now that

$$
\lim _{h \rightarrow \infty}\left\|T\left(y^{h, \delta}\right)\right\|_{p, q}=0
$$


In the other case, the coordinatewise null sequence $\left\{T\left(y^{h, \delta}\right)\right\}_{h=1}^{\infty}$ would have a seminormalized subsequence and there would be a further subsequence (again denoted by $\left.\left\{T\left(y^{h, \delta}\right)\right\}_{h=1}^{\infty}\right)$ such that Corollary 2.3 .1 holds. Since the strict inclusion $\ell^{q} \subset \ell^{u, v}$ holds, we take a strictly decreasing sequence $\left(\alpha_{h}\right) \in \ell^{u, v} \backslash \ell^{q}$. By the computations of (40) we obtain that the set $R_{0}:=\left\{\sum_{h=1}^{n} \alpha_{h} y^{h, \delta} \mid n \in \mathrm{N}\right\}$ is bounded in $\ell^{u, v}(v)$. However, by Corollary 2.3.1, $T\left(R_{0}\right)$ is unbounded in $\ell^{p, q}$, since $\left(\alpha_{h}\right) \notin \ell^{q}$. This proves our assertion.

As a consequence, if $s_{0} \in \mathrm{N}$ satisfies $\frac{1}{s_{0}}<\frac{\varepsilon}{2}$, for every $s \geq s_{0}$ we can determine inductively some $h_{s} \in \mathrm{N}$ such that $h_{s}>h_{s-1}$ and

$$
\forall s \geq s_{0}, \quad\left\|T\left(y^{h_{s}, \frac{1}{s}}\right)\right\|_{p, q}^{t} \leq \frac{1}{s} .
$$

Let us see now that the sequence

$$
\left\{\gamma^{s}\right\}_{s=s_{0}}^{\infty}:=\left\{\sum_{i \in P_{h_{s}, \frac{1}{s}}} z_{i}^{k_{h_{s}}} e_{i}\right\}_{s=s_{0}}^{\infty}
$$

has a seminormalized subsequence. If not then

$$
\lim _{s \rightarrow \infty}\left\|\gamma^{s}\right\|_{\ell^{u, v}(v)}=0 .
$$

Hence, by (42) and the continuity of $T$ it follows that $\left\{T\left(w^{h_{s}, \frac{1}{s}}\right)\right\}_{s=s_{0}}^{\infty}$ is a seminormalized coordinatewise null sequence in $\ell^{p, q}(\mu)$. Put $F_{s}:=\left\{i \in J_{h_{s}} \mid\right.$ $\left.i \notin P_{h_{s}, \frac{1}{s}} \cup G_{h_{s}, \frac{1}{s}}\right\}$ for every $s \geq s_{0}$ and $F:=\cup_{s=s_{0}}^{\infty} F_{s}$. By (38)

$$
\forall s \geq s_{0}, \quad M^{u} \geq\left\|\sum_{i \in F_{s}} z_{i}^{k_{h_{s}}} e_{i}\right\|_{u, v}^{u} \geq\left(\frac{u}{v}\right)^{\frac{u}{v}} s^{k u} \sum_{i \in F_{s}} v_{i}
$$

and hence

$$
v(F) \leq M^{u}\left(\frac{v}{u}\right)^{\frac{u}{v}} \sum_{s=s_{0}}^{\infty} \frac{1}{s^{k u}}<\infty .
$$

Now $\left\{w^{h_{s}, \frac{1}{s}}\right\}_{s=s_{0}}^{\infty}$ can be viewed as a sequence in the Lorentz sequence space $\ell^{u, v}(F, v)$ defined over the finite measure space $(F, v)$. By Lemma 4.2.b, it has a subsequence (again denoted by the same symbol), which is equivalent to the standard unit vector basis of $\ell^{v}$. By Corollary 2.3, we would get the existence of another subsequence (for which we retain the same notation) such that $\left\{T\left(w^{h_{s}, \frac{1}{s}}\right)\right\}_{s=s_{0}}^{\infty}$ satisfies (15). Choosing a sequence $\left(\alpha_{i}\right) \in \ell^{v} \backslash \ell^{q}$, the set $R_{1}:=\left\{\sum_{s=1}^{n} \alpha_{s} w^{h_{s}, \frac{1}{s}} \mid n \in \mathrm{N}\right\}$ is bounded in $\ell^{u, v}(v)$ while $T\left(R_{1}\right)$ is unbounded in $\ell^{p, q}(\mu)$ by Corollary 2.3.1. We have arrived at a contradiction 
which proves the existence of a seminormalized subsequence of $\left\{\gamma^{s}\right\}_{s=1}^{\infty}$ (again denoted by the same symbol).

By Theorem 2.4, for every number $0<\theta<1$, $\ell^{u, v}(v)$ is isomorphic with the interpolation space $\left(\ell^{(1-\theta) u}(v), \ell^{\infty}(v)\right)_{\theta, v}$. Since by construction

$$
\left\|z^{h_{s}}-y^{h_{s}, \frac{1}{s}}-w^{h_{s}, \frac{1}{s}}\right\|_{\ell^{\infty}(v)} \leq \frac{1}{s}
$$

by Theorem 2.6, switching to a suitable subsequence, we can assume $\left\{\gamma^{s}\right\}_{s=1}^{\infty}$, is equivalent to the canonical basis of $\ell^{v}$. As a consequence, since $\ell^{u, v} \subset \ell^{v}$, the set

$$
W:=\left\{\sum_{h=1}^{n} \alpha_{s} \gamma^{s} \mid\left\|\left(\alpha_{s}\right)\right\|_{\ell^{u, v}} \leq 1\right\}
$$

is bounded in $\ell^{u, v}(v)$. Then, arguing as in the previous case for the sequence $\left\{T\left(y^{h, \delta}\right)\right\}_{h=1}^{\infty}$, we can show that $\left\{T\left(\gamma_{s}\right)\right\}_{s=1}^{\infty}$, is a coordinatewise null sequence also. The proof is finished.

Proposition 4.4. Let $T \in \mathscr{L}\left(\ell^{u, v}(v), \ell^{p, q}(\mu)\right)$. Suppose that $\lim _{i \rightarrow \infty} \mu_{i}=$ $\infty$ or $\mu(\mathrm{N})<\infty$ and $q \leq u<v$. If $\left\{z^{n}\right\}_{n=1}^{\infty} \subset \ell^{u, v}(v)$ and $\left\{T\left(z^{n}\right)\right\}_{n=1}^{\infty} \subset$ $\ell^{p, q}(\mu)$ are coordinatewise null seminormalized sequences, there is a subsequence $\left\{\gamma^{n}\right\}_{n=1}^{\infty}$ of general sections $\left\{z^{n}\right\}_{n=1}^{\infty}$ which is equivalent to the standard unit basis of $\ell^{v}$ and such that $\left\{T\left(\gamma^{n}\right)\right\}_{n=1}^{\infty}$ is equivalent to the standard unit basis of $\ell^{q}$.

Proof. By Lemma 4.2.a or Lemma 4.2.b respectively, it is enough to find a subsequence $\left\{\gamma^{n}\right\}_{n=1}^{\infty}$ of general sections equivalent to the canonical unit basis of $\ell^{v}$ and such that $\left\{T\left(\gamma^{n}\right)\right\}_{n=1}^{\infty}$ is seminormalized and coordinatewise null in $\ell^{p, q}(\mu)$. The proof of this fact is exactly the same as the one given in Proposition 4.3 except for showing that (using the same notations of Proposition 4.3) $\lim _{s \rightarrow \infty}\left\|T\left(y^{s, \delta}\right)\right\|_{p, q}=0$. Now we argue as follows: If $\left\{T\left(y^{s, \delta}\right)\right\}_{s=1}^{\infty}$ has a seminormalized subsequence, by the gliding hump method used in Proposition 4.1 , we can choose a diagonal subsequence of consecutive linear sections $\left\{\omega^{m}\right\}_{m=1}^{\infty}:=\left\{T\left(y^{s_{m}, \delta}\right)\right\}_{m=1}^{\infty}$ satisfying the analogous inequalities of (28) and (29) for every $m \in N$. By Lemma 4.2.a or Lemma 4.2.b, $\left\{\omega^{m}\right\}_{m=1}^{\infty}$ has a subsequence (denoted by the same symbol) which is equivalent to the canonical unit basis of $\ell^{q}$. Now, taking an strictly decreasing sequence $\left(\alpha_{i}\right) \in \ell^{u, v} \backslash \ell^{u}$, the set $R_{1}:=\left\{\sum_{m=1}^{n} \alpha_{m} y^{s_{m}, \delta} \mid m \in \mathrm{N}\right\}$ is bounded in $\ell^{u, v}(v)$ by (40) but $T\left(R_{1}\right)$ is unbounded in $\ell^{q}$, since $\left(\alpha_{i}\right) \notin \ell^{q}$. From here on, we can argue as in Proposition 4.3 and get the desired subsequence.

Proposition 4.5. Let $\lim _{i \rightarrow \infty} v_{i}=\infty$ or $v(\mathrm{~N})<\infty$. Let $T$ be an operator from $\ell^{u, v}(v)$ into $\left.\ell^{p, q}(\mu)\right)$. If $\left\{z^{n}\right\}_{n=1}^{\infty} \subset \ell^{u, v}(v)$ and $\left\{T\left(z^{n}\right)\right\}_{n=1}^{\infty} \subset \ell^{p, q}(\mu)$ are 
coordinatewise null seminormalized sequences, there is a subsequence $\left\{u^{n}\right\}_{n=1}^{\infty}$ of $\left\{z^{n}\right\}_{n=1}^{\infty}$ which is equivalent to the canonical unit basis of $\ell^{v}$ and such that $\left\{T\left(u^{n}\right)\right\}_{n=1}^{\infty}$ is equivalent to the canonical unit basis of $\ell^{q}$ in following cases:

a) If $q \leq p<v$

b) If $q<p \leq v$

Proof. By Lemma 4.2.a or Lemma 4.2.b respectively, passing to a suitable subsequence, we assume that $\left\{z^{n}\right\}_{n=1}^{\infty}$ is equivalent to the standard unit basis of $\ell^{v}$. Now, retaining the notation of Proposition 4.1 concerning the sets $J_{h}$, components of every $z^{h}$ and the selection of the sequence $\left\{s_{h}\right\}_{h=1}^{\infty} \subset \mathrm{N}$, as the strict inclusion $\ell^{p, q} \subset \ell^{v}$ holds in the two cases a) and b), the proof of our proposition is exactly the same as that of Proposition 4.1 since now the set $\left\{\sum_{h=1}^{n} \alpha_{h} z^{s_{h}} \mid n \in \mathrm{N}\right\}$ is bounded in $\ell^{u, v}(v)$ whatever the strictly decreasing sequence $\left(\alpha_{i}\right) \in \ell^{v}$ may be (by the equivalence of $\left\{z^{n}\right\}_{n=1}^{\infty}$ with the canonical basis of $\ell^{v}$ ).

The crucial part of our main result will follow from a suitable application of previous propositions:

Theorem 4.6. Let $\ell^{u, v}(v)$ and $\ell^{p, q}(\mu)$ be Lorentz sequence spaces.

1) If $v$ and $\mu$ are measures $\rho=\left\{\rho_{i}\right\}_{i=1}^{\infty}$ such that $\rho(\mathrm{N})<\infty$ or $\lim _{i \rightarrow \infty} \rho_{i}=$ $\infty,\left(\ell^{u, v}(v), \ell^{p, q}(\mu)\right) \in \mathscr{K}$ if and only if $v>q$.

2) If $\nu(\mathrm{N})<\infty$ or $\lim _{i \rightarrow \infty} v_{i}=\infty$, and $\mu$ is a measure such that $\mu(\mathrm{N})=$ $\infty$ and $\lim _{i \rightarrow \infty} \mu_{i}=0$, or $\left\{\mu_{i}\right\}_{i=1}^{\infty}$ has an adherent point in $] 0, \infty[$, $\left(\ell^{u, v}(v), \ell^{p, q}(\mu)\right) \in \mathscr{K}$ if and only if $v>q$ and $v \geq p$.

3) If $v$ is a measure such that $v(\mathrm{~N})=\infty$ and $\lim _{i \rightarrow \infty} v_{i}=0$, or $\left\{v_{i}\right\}_{i=1}^{\infty}$ has an adherent point in ]0, $\infty$, and $\mu(\mathrm{N})<\infty$ or $\lim _{i \rightarrow \infty} \mu_{i}=\infty$, $\left(\ell^{u, v}(\nu), \ell^{p, q}(\mu)\right) \in \mathscr{K}$ if and only if $v>q$ and $u \geq q$.

4) If $v$ and $\mu$ are measures $\rho=\left\{\rho_{i}\right\}_{i=1}^{\infty}$ such that $\rho(\mathrm{N})=\infty$ and $\lim _{i \rightarrow \infty} \rho_{i}=$ 0 , or $\left\{\rho_{i}\right\}_{i=1}^{\infty}$ has an adherent point in $] 0, \infty\left[,\left(\ell^{u, v}(v), \ell^{p, q}(\mu)\right) \in \mathscr{K}\right.$ if and only if $v>q, v \geq p, u \geq p$ and $u \geq q$.

ProOf OF THE NECESSARY CONDITION. a) The necessity of condition $v>q$ in all cases follows from Lemma 3.1. The necessity of $u \geq p$ in the quoted cases follows from Lemma 3.2 and Lemma 3.3.

b) Suppose $v<p$ and that $\left\{\mu_{i}\right\}_{i=1}^{\infty}$ has an adherent point in $] 0, \infty[$. Arguing as in Lemma 3.2 and projecting onto a suitable complemented subspace, we may assume that $\ell^{p, q}(\mu)$ is isomorphic to $\ell^{p, q}$. By Proposition 2.7, $\ell^{v}$ is a complemented subspace of $\ell^{u, v}(v)$. Since the non compact inclusion $\ell^{v} \subset \ell^{p, q}$ holds, we have non compact maps from $\ell^{u, v}(v)$ into $\ell^{p, q}$. 
c) Suppose $\lim _{i \rightarrow \infty} \mu_{i}=0$ but $\mu(\mathrm{N})=\infty$ and $v<p$. By Lemma 3.3.a there is a non compact operator $T$ from $\ell^{v}$ into $\ell^{p, q}(\mu)$. We finish by composing $T$ with a continuous projection of $\ell^{u, v}(v)$ onto its subspace $\ell^{v}$ (Proposition 2.7).

d) Suppose that $\left\{v_{i}\right\}_{i=1}^{\infty}$ has an adherent point in ]0, $\infty$ [. Suppose moreover $u<q<v$ holds. Projecting onto a sectional subspace of $\ell^{u, v}(v)$, we can assume that $\ell^{u, v}(v)$ is isomorphic to $\ell^{u, v}$. Then we have the continuous non compact inclusion $I: \ell^{u, v} \subset \ell^{q, q}=\ell^{q}$. Since $\ell^{q}$ is a subspace of $\ell^{p, q}(\mu)$ (Proposition 2.7) there are non compact maps from $\ell^{u, v}(v)$ into $\ell^{p, q}(\mu)$.

e) Assume now that $v=\left(v_{i}\right)$ is a measure on $\mathrm{N}$ such that $\lim _{i \rightarrow \infty} v_{i}=0$ but $v(\mathrm{~N})=\infty$. Also assume $u<q<v$. By Lemma 3.3.b there is a non compact map from $\ell^{u, v}(v)$ into $\ell^{q, q}=\ell^{q}$. Since $\ell^{q}$ is a topological subspace of $\ell^{p, q}(\mu)$ (Proposition 2.7), we have finished.

Proof of THE SUFFICIENT CONDITION. Let $T$ be an operator from $\ell^{u, v}(v)$ into $\ell^{p, q}(\mu)$. We need to show that $\left\{T\left(z^{n}\right)\right\}_{n=1}^{\infty}$ has a convergent subsequence for every bounded sequence $\left\{z^{n}\right\}_{n=1}^{\infty}=\left\{\left(z_{i}^{n}\right)\right\}_{n=1}^{\infty} \subset \ell^{u, v}(v)$. As the projections onto each axis are continuous, by a diagonalization procedure we can select a subsequence, again denoted by $\left\{z^{n}\right\}_{n=1}^{\infty}$, such that

$$
\forall i \in \mathrm{N}, \quad \lim _{n \rightarrow \infty} z_{i}^{n} \text { exists. }
$$

Since every map $P_{n} T, n \in \mathrm{N}$, is compact, another application of the diagonalization procedure, we can choose a subsequence, still denoted by $\left\{z^{n}\right\}$, such that $\left\{\left(w_{i}^{n}\right)\right\}_{n=1}^{\infty}:=\left\{T\left(\left(z_{i}^{n}\right)\right)\right\}_{n=1}^{\infty}$ is coordinatewise convergent. The theorem will be shown if we prove that $\left\{\left(w_{i}^{n}\right)\right\}_{n=1}^{\infty}$ is a Cauchy sequence in $\ell^{p, q}(\mu)$. By a useful and well known observation due to Pelcynski (see [15]) and (44), it is enough to see that $\lim _{n \rightarrow \infty} T\left(z^{n}\right)=0$ in $\ell^{p, q}(\mu)$ for every bounded coordinatewise null sequence $\left\{z^{n}\right\}_{n=1}^{\infty} \subset \ell^{u, v}(\nu)$ such that $\left\{\left(w_{i}^{n}\right)\right\}_{n=1}^{\infty}$ is also coordinatewise null in $\ell^{p, q}(\mu)$.

Suppose that $\left\{T\left(z^{n}\right)\right\}_{n=1}^{\infty}$ does not converge to 0 . Then we can choose a seminormalized subsequence (again denoted by $\left\{T\left(z^{n}\right)\right\}_{n=1}^{\infty}$ ). A fortiori, by the continuity of $T,\left\{\left(z^{n}\right)\right\}_{n=1}^{\infty}$ will be seminormalized also in $\ell^{u, v}(v)$. From now on we consider seven cases which covers all possibilities:

Case I. Suppose $s:=\max \{p, q\}<r:=\min \{u, v\}$. Choose a sequence $\left(\alpha_{i}\right) \in \ell^{r}$ such that $\left(\alpha_{i}\right) \notin \ell^{s}$. By a successive application of Corollary 2.3.2 and Corollary 2.3.1 we get a subsequence $\left\{z^{k_{m}}\right\}_{m=1}^{\infty}$ such that the set $R:=$ $\left\{\sum_{m=1}^{h} \alpha_{m} z^{k_{m}} \mid h \in \mathrm{N}\right\}$ is bounded in $\ell^{u, v}(v)$ but $T(R):=\left\{\sum_{m=1}^{h} \alpha_{m} T\left(z^{k_{m}}\right) \mid\right.$ $h \in \mathrm{N}\}$ is unbounded in $\ell^{p, q}(\mu)$, contradicting the continuity of $T$.

Case II. Suppose $q<p \leq r=\min \{u, v\}$. By Proposition 4.1, by replacing $\left\{z^{n}\right\}_{n=1}^{\infty}$ with a subsequence if necessary, we can suppose that $\left\{T\left(z^{n}\right)\right\}_{n=1}^{\infty}$ is 
equivalent to the standard unit basis of $\ell^{q}$. Choosing a sequence $\left(\alpha_{i}\right) \in \ell^{r}$ such that $\left(\alpha_{i}\right) \notin \ell^{q}$, we obtain a contradiction as above by Corollary 2.3.2.

Case III. Suppose $p \leq q \leq u<v$. Arguing as in Corollary 2.3 (gliding hump method), we can find a diagonal subsequence $\left\{u^{m}\right\}_{m=1}^{\infty}$ of consecutive linear sections of $\left\{z^{n}\right\}_{n=1}^{\infty}$ satisfying the inequalities (20), (21) and (22). These inequalities, the continuity of every projection $P_{i}, i \in \mathrm{N}$, the actual hypothesis on $\left\{T\left(z^{n}\right)\right\}_{n=1}^{\infty}$ and (17) imply that $\left\{T\left(u^{m}\right)\right\}_{m=1}^{\infty}$ is again a seminormalized coordinatewise null sequence in $\ell^{p, q}(\mu)$. By Proposition 4.3, $\left\{u^{m}\right\}_{m=1}^{\infty}$ has a diagonal subsequence $\left\{\gamma^{m_{s}}\right\}_{s=1}^{\infty}$ of general sections which is equivalent to the standard unit basis of $\ell^{v}$ and such that $\left\{T\left(\gamma^{m_{s}}\right)\right\}_{s=1}^{\infty}$ is a coordinatewise null semi-normalized sequence in $\ell^{p, q}(\mu)$. Selecting a suitable subsequence, we may assume that $\left\{T\left(\gamma^{m_{s}}\right)\right\}_{s=1}^{\infty}$ satisfies Corollary 2.3.1. Taking a sequence $\left(\alpha_{i}\right) \in \ell^{v} \backslash \ell^{q}$ we finish the proof as in case II, using this Corollary.

Case IV. Suppose that $\lim _{i \rightarrow \infty} v_{i}=\infty$, and $q<v$. By Lemma 4.2.a, looking at a suitable subsequence, we may assume that $\left\{z^{m}\right\}_{m=1}^{\infty}$ is equivalent to the canonical unit basis of $\ell^{v}$. Clearly $\left\{T\left(z^{m}\right)\right\}_{m=1}^{\infty}$ is again a coordinatewise null seminormalized sequence in $\ell^{p, q}(\mu)$.

a) Suppose moreover that $\lim _{i \rightarrow \infty} \mu_{i}=\infty$ or $\mu(\mathrm{N})<\infty$. If $p \leq q$, $\left\{T\left(z^{m}\right)\right\}_{m=1}^{\infty}$ has a subsequence such that Corollary 2.3.1 holds. Then we finish the proof as above choosing a sequence $\left(\alpha_{i}\right) \in \ell^{v} \backslash \ell^{q}$. In the case $q<p$, by Lemma 4.2.a and Lemma 4.2.b respectively, $\left\{T\left(z^{m}\right)\right\}_{m=1}^{\infty}$ has a subsequence equivalent to the canonical unit basis of $\ell^{q}$. We get a contradiction by choosing a sequence $\left(\alpha_{i}\right) \in \ell^{v} \backslash \ell^{q}$.

b) Now suppose $p \leq v$. If $p \leq q$, since $q<v$, we argue as in case IV.a. If $q<p \leq v$, by Proposition 4.5, $\left\{z^{m}\right\}_{m=1}^{\infty}$ can be chosen indeed such that $\left\{T\left(z^{m}\right)\right\}_{m=1}^{\infty}$ is equivalent to the canonical basis of $\ell^{q}$. We finish by choosing $\left(\alpha_{i}\right) \in \ell^{v} \backslash \ell^{q}$.

Case V. Suppose $v(\mathrm{~N})=\infty$ but $\lim _{i \rightarrow \infty} v_{i}=0$ and $v>q, u \geq q$.

a) Let $\lim _{i \rightarrow \infty} \mu_{i}=\infty$. If $v \leq u$, by Corollary 2.3.2. and by Lemma 4.2.a, by switching to a suitable subsequence, we may assume that $\left\{z^{m}\right\}_{m=1}^{\infty}$ satisfies Corollary 3.2 and $\left\{T\left(z^{m}\right)\right\}_{m=1}^{\infty}$ is equivalent to the standard unit basis of $\ell^{q}$. Then we use a sequence $\left(\alpha_{i}\right) \in \ell^{q} \backslash \ell^{v}$ and Corollary 2.3.2.

If $q \leq u<v$ by Proposition $4.4,\left\{z^{m}\right\}_{m=1}^{\infty}$ has a subsequence $\left\{\gamma^{m}\right\}_{m=1}^{\infty}$ of diagonal general sections which is equivalent to the canonical unit basis of $\ell^{v}$ and $\left\{T\left(\gamma^{m}\right)\right\}_{m=1}^{\infty}$ is equivalent to the canonical unit basis of $\ell^{q}$. Then we finish as above using the same sequence $\left(\alpha_{i}\right)$.

b) If $\mu(\mathrm{N})<\infty$, the proof is analogous to case V.a using Lemma 4.2.b and Corollary 2.3.2 when $q<v \leq u$, and Proposition 4.4 and Lemma 4.2.b if $q \leq u<v$

Case VI. Suppose $v(\mathrm{~N})<\infty$ and $v>q$. 
a) Let $\mu(\mathrm{N})<\infty$. By consecutive applications of Lemma 4.2.b in $\ell^{u, v}(v)$ and $\ell^{p, q}(\mu)$, choosing a suitable subsequence, we can assume that $\left\{z^{m}\right\}_{m=1}^{\infty}$ is equivalent to the canonical unit vector basis of $\ell^{v}$ and $\left\{T\left(z^{m}\right)\right\}_{m=1}^{\infty}$ is equivalent to the canonical unit vector basis of $\ell^{q}$. Then we finish in the known way using a sequence $\left(\alpha_{i}\right) \in \ell^{v} \backslash \ell^{q}$.

b) If $\lim _{i \rightarrow \infty} \mu_{i}=\infty$, the proof is the same using Lemma 4.2.a.

c) Suppose now $v>q, v \geq p$. If moreover $q \leq p<v$ or $q<p \leq v$ holds, by Proposition 4.5, selecting suitable subsequences, we may assume that $\left\{z^{m}\right\}_{m=1}^{\infty}$ and $\left\{T\left(z^{m}\right)\right\}_{m=1}^{\infty}$ are equivalent to the standard unit bases of $\ell^{v}$ and $\ell^{q}$ respectively. We finish using a sequence $\left(\alpha_{i}\right) \in \ell^{v} \backslash \ell^{q}$. Analogously, if $p \leq q<v$ holds, by Lemma 4.2.b and Corollary 2.3.1, we can assume that $\left\{z^{m}\right\}_{m=1}^{\infty}$ is equivalent to the canonical unit basis of $\ell^{v}$ and $\left\{T\left(z^{m}\right)\right\}_{m=1}^{\infty}$ satisfies Corollary 2.3.1. Then we finish with the same $\left(\alpha_{i}\right)$ in the customary way.

Case VII. To finish, suppose that $v>q, u \geq q$, the measure $v$ is such that the sequence $\left\{v_{i}\right\}_{i=1}^{\infty}$ has an adherent point in the open interval $] 0, \infty[$, and $\lim _{i \rightarrow \infty} \mu_{i}=\infty$ or $\mu(\mathrm{N})<\infty$. Now the proof is the same as in case V.b using Lemma 4.2.a and Lemma 4.2.b respectively.

\section{REFERENCES}

1. Beauzamy, B., Espaces d'Interpolation Réels: Topologie et Géometrie, Lecture Notes in Math. 666 (1978).

2. Bennet, C., Sharpley, R., Interpolation of Operators, Pure Appl. Math. 129, Academic Press, Boston (1988).

3. Brudnyi, Yu. A., Krugljak, N. Ya., Interpolation functors and interpolation spaces, Vol. 1, North-Holland Math. Library 47 (1991).

4. Castillo, J., López Molina, J. A., Operators defined on projective and natural tensor products, Michigan Math. J. 40 (1993), 411-415.

5. Defant, A., López Molina, J. A., Rivera, M. J., On Pitt's theorem for operators between quasi Banach sequence spaces, Monatsh. Math. 130 (2000), 7-18.

6. Freitag, D., Real interpolation of weighted $L_{p}$-spaces, Math. Nachr. 86 (1978), 15-18.

7. Kalton, N. J., Peck, N. T., Roberts, W., An F-space sampler, London Math. Soc. Lecture Note Ser. 89 (1984).

8. Kalton, N. J., Montgomery-Smith, S. J., Set-functions and factorization, Arch. Math. 61 (1993), 183-200.

9. Levy, M., L'espace d' interpolation réel $\left(A_{0}, A_{1}\right)_{\theta, p}$ contient $\ell_{p}$, C. R. Acad. Sci. Paris, Ser. A 289 (1979), 675-677.

10. Lindenstrauss, J., Tzafriri, L., Classical Banach Spaces II, Springer Verlag, Berlin, Heidelberg, New York, 1979.

11. López Molina, J.A., On subspaces isomorphic to $\ell^{q}$ in interpolation of quasi Banach spaces, Turkish J. Math. 24 (2000), 373-378.

12. López Molina, J. A., Existence of complemented subspaces isomorphic to $\ell^{q}$ in interpolation of quasi Banach spaces, preprint (2000).

13. López Molina, J. A., Rivera, M. J., Bounded, compact and Montel maps on projective tensor products of Köthe spaces, Bull. Polish Acad. Sci. Math. 44, 4 (1996), 411-426.

14. Pietsch, A., Eigenvalues and s-numbers, Cambridge University Press, Cambridge, 1987. 
15. Rosenthal, H., Quasicomplemented subspaces of Banach spaces with an appendix on compact operators on $L^{p}$, J. Funct. Anal. 4 (1969), 176-214.

16. Sagher, Y., Interpolation of $r$-Banach spaces, Studia Math. 41, 1 (1972), 45-70.

E.T.S. INGENIEROS AGRÓNOMOS

CAMINO DE VERA

46023 VALENCIA

SPAIN

E-mail: jalopez@mat.upv.es 